\title{
Effect of Bedding Planes on the Permeability and Diffusivity Anisotropies of Berea Sandstone
}

\author{
Minoru Sato $^{1}$ D $\cdot$ Kamran Panaghi $^{2} \cdot$ Naoki Takada $^{1} \cdot$ Mikio Takeda $^{1}$
}

Received: 3 April 2018 / Accepted: 28 November 2018 / Published online: 10 December 2018

(C) The Author(s) 2018

\begin{abstract}
Evaluating the anisotropy of transport parameters in rocks is important for various applications, such as reservoir engineering and rock mechanics. Owing to their anisotropic pore structures, the tortuosity, constrictivity, and pore size distribution of rocks are often anisotropic in nature, which in turn affect the permeability and diffusivity. However, it has still not been determined whether the permeability and diffusivity are anisotropic in the same manner. This study used experiments and numerical modeling to examine the effect of the pore structure on the permeability and diffusivity anisotropies of rocks. The experimental results showed a clear difference in the anisotropy ratios of the permeability $\left(k^{\perp} / k^{\|}\right)$and diffusivity $\left(D_{\mathrm{e}}^{\perp} / D_{\mathrm{e}}^{\|}\right)$for Berea sandstone, which is the de facto standard porous sandstone. The analysis results from micro-focus X-ray computed tomography and simulation with the lattice Boltzmann method supported the experimental difference in anisotropy ratios. In the analysis and simulation, the relation between the minimum cross-sectional porosity area and characteristic pressure gradient was estimated. The analysis results suggest that the minimum cross-sectional porosity areas that influence the permeability anisotropy are too large to physically induce anisotropic $\mathrm{NaCl}$ diffusion, and thus, the diffusivity of Berea sandstone is nearly isotropic.
\end{abstract}

Keywords Permeability $\cdot$ Diffusivity $\cdot$ Anisotropy $\cdot$ Berea sandstone $\cdot$ Bottleneck effect

\section{Introduction}

Studying the transport parameters of rocks in deep underground structures is required for geological disposal and/or reservoir engineering applications. Cases in point are measures to hinder the migration of radioactive waste from geological repositories to the biosphere and storing $\mathrm{CO}_{2}$ in porous subsurface formations. Accordingly, subsurface rock formations are of

Minoru Sato

minoru-satou@aist.go.jp

1 AIST (National Institute of Advanced Industrial Science and Technology), 1-1-1 Higashi, Tsukuba, Ibaraki, Japan

2 Civil Engineering Department, University of Kurdistan Hewlêr, 30-Meter Avenue, Erbil, Kurdistan Region, Iraq 
interest to rock mechanics experts as they can potentially be viable sites for the development of underground structures.

Several studies have indicated that anisotropy in the pore structure and intermittent connectivity cause anisotropy in the domain's permeability and diffusivity. In sandstone, for instance, the permeability in the direction parallel to the bedding is controlled by the highly permeable clay-free layers while the permeability in the direction normal to the bedding is determined by the shale content of the less permeable silty layers (Clavaud et al. 2008). Farver and Yund (1999) reported that the diffusion coefficient of oxygen in the direction parallel to foliation for a mica-bearing rock is approximately 2-10 times greater than that in the direction normal to the bedding. The diffusivity of clay samples after compaction has also been observed to be much higher in the direction parallel to the fabric than that normal to the fabric because of the lower tortuosity (Van Loon et al. 2004). Wan et al. (2015) demonstrated the same relation between the pore structure and diffusivity anisotropy of a single cubic shale sample by eliminating the impact of the samples' heterogeneity on the results. Yokoyama and Nakashima (2005) reported that the diffusion coefficients of pore water $\mathrm{K}^{+}$ ions in rhyolite were 5-6 and 7-9 times smaller in the direction normal to the flow plane than the other orthogonal and parallel orientations. Because the nuclides dissolved in water travel easily through the sufficiently large pore spaces in sandstone, advective flow takes on a significant role in their migration through groundwater. However, the advective flow is impeded by small pores or pore throat sizes, such as those in mudstone and shale. Consequently, diffusion becomes a relatively dominant phenomenon for nuclide migration through these materials. To determine the migration of substances such as dissolved nuclides and $\mathrm{CO}_{2}$ that depend on the flow state of groundwater, the anisotropic ratios for both the permeability and diffusivity are required. However, it is still unclear whether a transport parameter for either the permeability or diffusivity is applicable to the other.

In this study, both experimental procedure and numerical modeling were employed to examine the effects of the pore structure on the anisotropy of transport parameters for Berea sandstone, whose grain arrangement is dominated by sedimentation. To estimate the permeability and diffusion parameters, this work focused on the phenomena separately. Permeability and diffusivity experiments were performed on a single rock sample in sequence under an isotropic confining pressure. The experimental results indicated the anisotropy ratios for the permeability and diffusivity of Berea sandstone. To reveal the cause of the difference in anisotropy ratios, a numerical analysis was performed by using the pore geometry. The pore structure of Berea sandstone was examined with 3-D X-ray computed tomography (CT) to investigate the influence of the porosity, tortuosity, and constrictivity on the permeability and diffusivity of Berea sandstone. The diffusivity of the sample was calculated from the CT data, and its permeability was estimated with the lattice Boltzmann method (LBM). In computational fluid dynamics, LBM is a parallel and efficient algorithm that serves as a substitute for the Navier-Stokes equations (Benzi et al. 1992). LBM has been employed in various fields, including earth sciences and geology, to understand transport in open pores and porous structures. For example, it was used by Auzerais et al. (1996) to examine the scale dependence and influence of heterogeneities of transport properties in Fontainebleau sandstone; by Okabe and Blunt (2004) to predict the permeability of porous media based on 3-D CT images and reconstructed 2-D images; by Gao et al. (2015) to study the permeability of sandstones for the voxel size dependency; and by Genty et al. (2017) to study the microfracture of indurated argillite Oparinus clay and obtain the effective diffusion coefficient. Finally, the experimental and numerical results were compared to explore the relationship between the anisotropic transport parameters and bedding plane direction. 
Fig. 1 Berea sandstone for the permeability and diffusivity tests cored in the direction parallel to the bedding

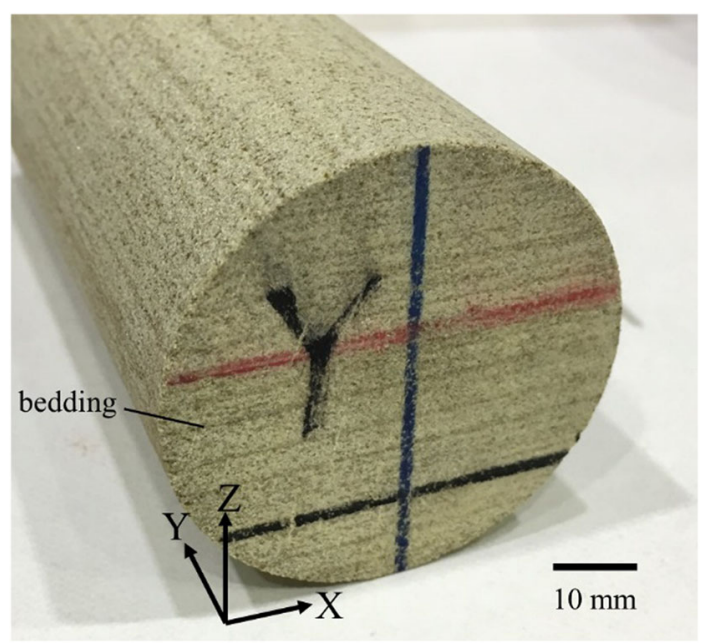

\section{Materials and Methods}

\subsection{Specimen Description}

Berea sandstone has long been used as the de facto standard material in laboratory experiments for rock mechanics and reservoir engineering applications, such as radioactive waste disposal, $\mathrm{CO}_{2}$ storage, or petroleum engineering practices. For permeability and diffusivity tests, diskshaped rock samples with a diameter of $50 \mathrm{~mm}$ and thickness of $10 \mathrm{~mm}$ were extracted in two orthogonal directions from a block, with the $Z$ direction being normal to the bedding plane and the $X$ and $Y$ directions being parallel to that (Fig. 1). The Berea sandstone used in the study was comprised of predominantly well-sorted and well-rounded quartz grains with minor amounts of feldspar and opaque minerals (Fig. 2). The bedding was easily visible and consisted of opaque minerals (iron sand and minor amounts of zircon). The porosity of the bedding bands is about lower than that of the other parts of the sandstone, and this was observed using X-ray CT data, as described in Sect. 3.2. The characteristic lamination showed that the Berea sandstone was deposited as beach sediments (Fujii et al. 2015). The porosity, pore structure, and average pore radius of the samples were characterized by mercury intrusion porosimetry using a Micrometrics AutoPore IV 9520 to determine the pore size distribution from 2 to $500 \mu \mathrm{m}$ (Fig. 3). The Berea sandstone had unimodal characteristics at 5-10 $\mu \mathrm{m}$, and small pores were present up to $5 \mathrm{~nm}$. The porosity was $18.29 \%$.

\subsection{Permeability Experiment}

The experimental system presented by Takeda et al. (2014) was employed to perform permeability and diffusivity experiments on a rock sample (Fig. 4). Prior to the permeability experiments, a rock sample was jacketed with a neoprene rubber and placed in the pressure vessel. The sample was immersed in a $0.1 \mathrm{M} \mathrm{NaCl}$ solution, which was used as the permeant fluid. The experiments were performed with the constant head method at a hydraulic head difference of $3 \mathrm{kPa}$ and a confining pressure of $500 \mathrm{kPa}$ (ASTM 2006) at room temperature $\left(25^{\circ} \mathrm{C}\right)$. The cumulative change in the effluent solution was measured by using an electric 
Fig. 2 Thin section of Berea sandstone. $Q t$ quartz, $F l$ feldspar, $O p$ opaque minerals

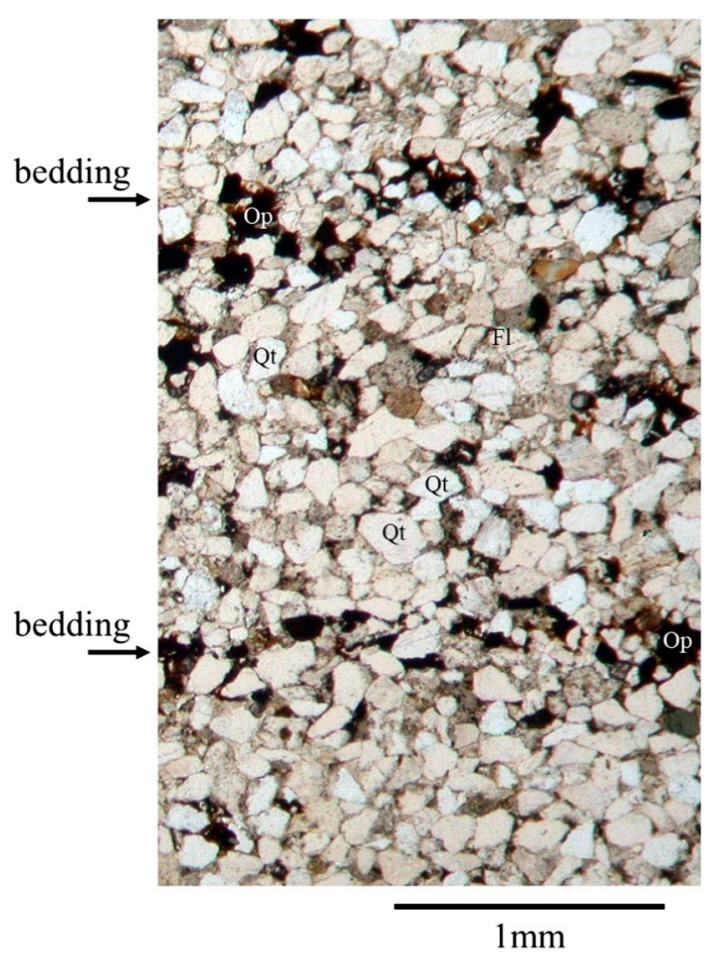

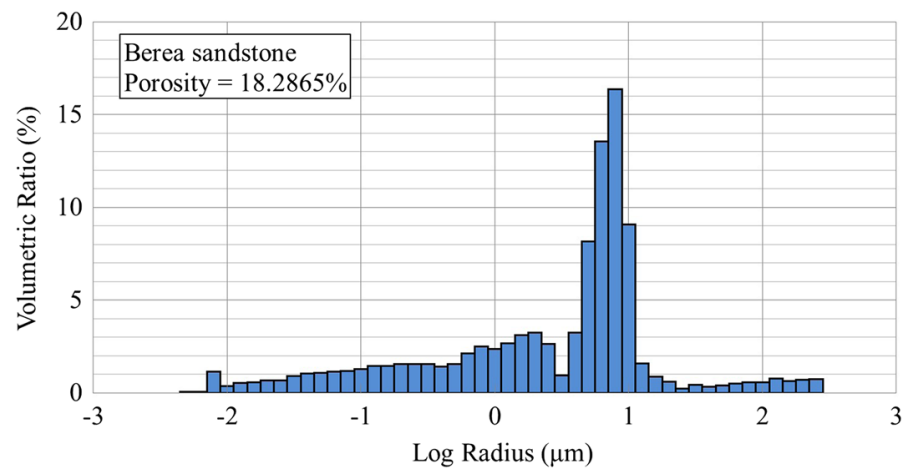

Fig. 3 Total porosity and pore size distribution of Berea sandstone with the mercury intrusion porosimeter

balance. The hydraulic conductivity $K(\mathrm{~m} / \mathrm{s})$ for the constant head experiment was obtained using Darcy's law:

$$
K=\frac{q L}{A H}
$$

where $q$ is the discharge $\left(\mathrm{m}^{3} / \mathrm{s}\right), L$ is the sample length $(\mathrm{m}), A$ is the cross-sectional area of the specimen $\left(\mathrm{m}^{2}\right)$, and $H$ is the constant head pressure $(\mathrm{m})$. The hydraulic conductivity can be converted into the intrinsic permeability $k\left(\mathrm{~m}^{2}\right)$ as follows:

$$
k=\frac{\eta}{\rho g} K
$$




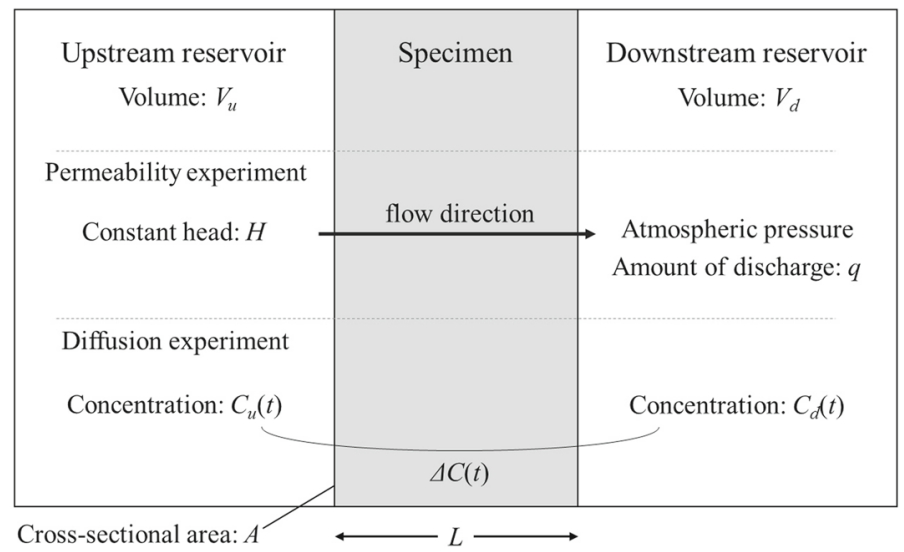

Fig. 4 Diagram for measuring the permeability and diffusivity of the sample

where $\rho$ and $\eta$ are the fluid density $\left(\mathrm{kg} / \mathrm{m}^{3}\right)$ and fluid viscosity (Pa s), respectively, and $g$ is the gravitational acceleration $\left(\mathrm{m} / \mathrm{s}^{2}\right)$. The permeability experiments were conducted at room temperature $\left(25^{\circ} \mathrm{C}\right)$, and $\rho$ and $\eta$ were $1.036 \times 10^{3} \mathrm{~kg} / \mathrm{m}^{3}$ and $9.6 \times 10^{-4} \mathrm{~Pa}$ s, respectively.

\subsection{Diffusion Experiment}

Diffusivity experiments were performed on each sample with the through-diffusion method at a concentration difference of $0.6-0.1 \mathrm{M} \mathrm{NaCl}$ and a confining pressure of $500 \mathrm{kPa}$. The temperature was controlled at $40{ }^{\circ} \mathrm{C}$ by using a heating system. This temperature was selected because it is difficult to keep the room temperature constant for 1 month as the air conditioner introduces errors of $\pm 1.5^{\circ} \mathrm{C}$. The reservoir solution was circulated by magnetic gear pumps to keep the solute concentration uniform in the reservoirs. The effective diffusion $D_{\mathrm{e}}$ can be calculated as follows (Wolfrum et al. 1988):

$$
\ln \left(\frac{\Delta C}{\Delta C_{0}}\right)=-\frac{A \cdot D_{\mathrm{e}}}{L} \cdot\left(\frac{1}{V_{\mathrm{d}}}+\frac{1}{V_{\mathrm{u}}}\right) \cdot t
$$

where $\Delta C$ and $\Delta C_{0}$ are the concentration differences between the upstream and downstream reservoirs at time $t$ and the initial concentration difference, respectively, and $V_{\mathrm{u}}$ and $V_{\mathrm{d}}$ correspondingly represent the upstream and downstream volumes of the reservoir. The temperature of the incubator was set to $40{ }^{\circ} \mathrm{C}$ for all tests.

\subsection{CT System for Micro-focus X-rays}

The 3-D geometry of Berea sandstone was captured using micro-focus X-ray CT with the lattice Boltzmann method to determine the permeability and diffusivity in the simulations. High-resolution X-ray CT was performed on a drilled cylindrical core with a diameter of $10 \mathrm{~mm}$. Each voxel of this 3-D image had a linear dimension of $5 \mu \mathrm{m}$. The size of the reconstructed 3-D image for the cube was $300^{3}$ voxels $\left(1.5 \times 1.5 \times 1.5 \mathrm{~mm}^{3}\right)$, and this included one of the sequential beddings on the $X-Y$ plane in the representative specimen. The cube of $300^{3}$ voxels represents the limit size of the LBM simulation by a standard computer (Intel 


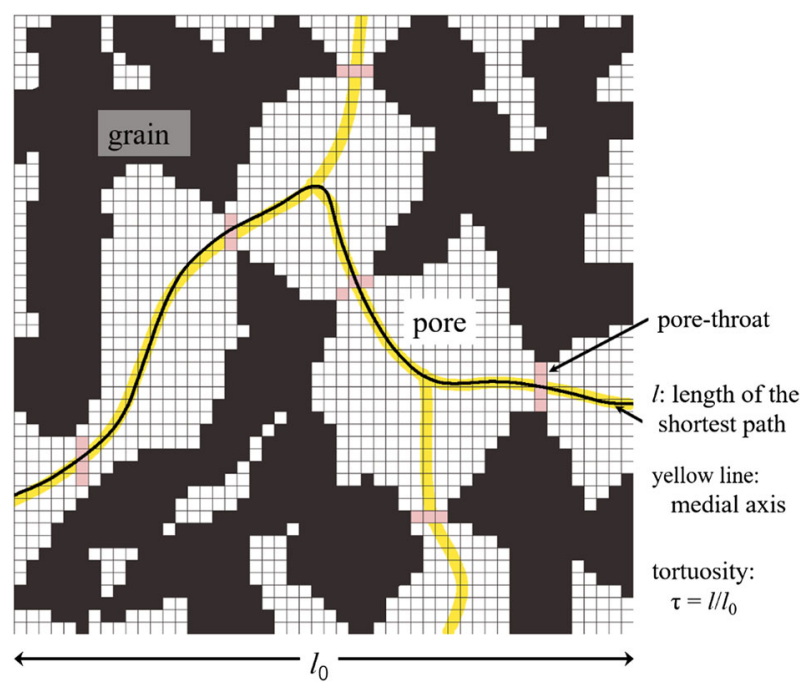

Fig. 5 Schematic of tortuosity of the porous matrix. The medial axis, shown in yellow, was obtained by applying the burn algorithm to the CT image

core i7 CPU x980 3.3 GHz (12 CPUs), 48 GB RAM). In general, CT images of porous media are gray scale and have a bimodal population: One mode corresponds to the signal from the pore space, and the other corresponds to that of the grain. We used the porosity from the results of the mercury intrusion porosimetry to binarize the samples into pore space and grain segments. The Kriging-based algorithm developed by Linquist and Venkatarangen (1999) was used to binarize the voxel images. By distinguishing the pore throat as a local minimum of the boundary sectional area for the pores (Fig. 5), data on the pore structure, spatial distribution for the pore and pore throat sizes, tortuosity, and porosity distribution were derived. The minimum pore throat size is $5 \mu \mathrm{m}$, which is restricted owing to the CT resolution. The medial axes of the pore structure were also obtained by using the burn algorithm (Linquist et al. 1996) and can be used to calculate the shortest paths and tortuosity from one end face to the opposite side (Fig. 5).

\subsection{Diffusion Modeling}

According to Van Brakel and Heertjes (1974), the effective diffusion coefficient $\left(D_{\mathrm{e}}\right)$ can be described in terms of the porosity $(\varphi)$, geometric constrictivity $\left(\delta_{\mathrm{g}}\right)$, tortuosity $(\tau)$, and tracer diffusivity in bulk liquid water, $D_{\mathrm{w}}\left(\mathrm{m}^{2} \mathrm{~s}^{-1}\right)$ :

$$
D_{\mathrm{e}}=\varphi \frac{\delta_{\mathrm{g}}}{\tau^{2}} D_{\mathrm{w}}
$$

The tortuosity is defined by

$$
\tau=l / l_{0}
$$

where $l_{0}$ is the apparent length of the element and $l$ is the length of the fluid flow path through the porous matrix (Fig. 5). As the tortuosity increases, the porous matrix becomes more complicated. When the fluid flow in a porous matrix is being considered, the tortuosity becomes meaningful for permeability. The constrictivity is generally the ratio of the radius 
of the diffusing particle to the pore radius; hence, it is a dimensionless parameter between 0 and 1. Takahashi et al. (2009) gave the following relation for the geometric constrictivity $\delta_{\mathrm{g}}$

$$
\delta_{\mathrm{g}}=\frac{(\text { maximum } \times \text { minimum cross-section })^{\frac{1}{2}}}{\text { mean cross section }}=\frac{\left(\pi r_{\mathrm{p}}^{2} \times \pi r_{\mathrm{t}}^{2}\right)^{\frac{1}{2}}}{\left(\frac{\pi r_{\mathrm{p}}^{2}+\pi r_{\mathrm{t}}^{2}}{2}\right)}
$$

where $r_{\mathrm{p}}$ and $r_{\mathrm{t}}$ are assumed to be the approximation radii of the pore and throat, respectively. In this study, $r_{\mathrm{p}}$ and $r_{\mathrm{t}}$ were derived from burn algorithm applied to the 3-D CT pore volume. $\delta_{\mathrm{g}}^{\|}$and $\delta_{\mathrm{g}}^{\perp}$ denote the average value of $\delta_{\mathrm{g}}$ through all shortest paths parallel and normal to the bedding plane.

\subsection{Flow Simulation with the Lattice Boltzmann Method (LBM)}

In the LBM, the evolution of the particle distribution function $f_{a}(\boldsymbol{x}, t)$ at point $\boldsymbol{x}$ and time $t$ with velocity $\boldsymbol{e}_{a}$ is computed as follows (Chen and Doolen 1998):

$$
\begin{gathered}
\frac{\partial f_{a}}{\partial t}=-\boldsymbol{e}_{a} \cdot \nabla f_{a}+\Omega_{a}(\boldsymbol{x}, t) \\
\Omega_{a}(\boldsymbol{x}, t)=-\frac{1}{\tau_{f}}\left[f_{a}(\boldsymbol{x}, t)-f_{a}^{\mathrm{eq}}(\boldsymbol{x}, t)\right]
\end{gathered}
$$

where $f_{a}^{\mathrm{eq}}$ is an equilibrium distribution function, $\tau_{f}$ is a single relaxation time, and $t$ is a time step during which the particle travels the lattice spacing. The Bhatnagar-Gross-Krook (BGK) model is used for the collision term $\Omega_{a}(x, t)$ in Eq. (7). According to the kinetic theory of fluids, the density $\rho$ and flow velocity $\boldsymbol{u}$ under a constant temperature can be defined in terms of the particle distribution function (Chen and Doolen 1998; Misawa et al. 2004):

$$
\begin{gathered}
\rho=\sum_{a} f_{a}=\sum_{a} f_{a}^{\mathrm{eq}} \\
\rho \boldsymbol{u}=\sum_{a} f_{a} \boldsymbol{e}_{a}=\sum_{a} f_{a}^{\mathrm{eq}} \boldsymbol{e}_{a}
\end{gathered}
$$

The D3Q15 model (Inamuro et al. 1999) for the particle velocity set was used in the following calculations. The Navier-Stokes equations have second-order nonlinearity. The general form of the equilibrium distribution function up to the second-order term can be written as (Chen and Doolen 1998)

$$
f_{a}^{\mathrm{eq}}=w_{a} \rho\left[1+\frac{\boldsymbol{e}_{\boldsymbol{a}} \cdot \boldsymbol{u}}{c_{s}^{2}}+\frac{\left(\boldsymbol{e}_{\boldsymbol{a}} \cdot \boldsymbol{u}\right)^{2}}{2 c_{s}^{4}}-\frac{\boldsymbol{u} \cdot \boldsymbol{u}}{2 c_{s}^{2}}\right]
$$

where $w_{a}=2 / 9(a=0), 1 / 9(a=1-6)$, and 1/72 $(a=7-14)$. This expansion is valid only for small velocities or small Mach numbers $|\boldsymbol{u}| / c_{s}$, where $c_{s}$ is the speed of sound. Equation (11) is applicable for flow with low compressibility. In the case of an incompressible flow ( $\rho_{0}$ is constant), $f_{a}^{\mathrm{eq}}$ is derived as follows (He and Luo 1997, Onishi et al. 2001):

$$
f_{a}^{\mathrm{eq}}=w_{a}\left[\frac{p}{c_{s}^{2}}+\rho_{0}\left\{\frac{\boldsymbol{e}_{\boldsymbol{a}} \cdot \boldsymbol{u}}{c_{s}^{2}}+\frac{\left(\boldsymbol{e}_{\boldsymbol{a}} \cdot \boldsymbol{u}\right)^{2}}{2 c_{s}^{4}}-\frac{\boldsymbol{u} \cdot \boldsymbol{u}}{2 c_{s}^{2}}\right\}\right]
$$


where $p$ is the pressure and $c_{s}=1 / \sqrt{3}$. The pressure and flow velocity are defined as follows:

$$
\begin{gathered}
p=\sum_{a} f_{a} c_{s}^{2}=\sum_{a} f_{a}^{\mathrm{eq}} c_{s}^{2} \\
\rho_{0} \boldsymbol{u}=\sum_{a} f_{a} \boldsymbol{e}_{a}=\sum_{a} f_{a}^{\mathrm{eq}} \boldsymbol{e}_{a}
\end{gathered}
$$

Equations (7) and (8) can be discretized into the semi-Lagrangian form with second-order accuracies in both space and time as follows:

$$
f_{a}\left(\boldsymbol{x}+\boldsymbol{e}_{a} \Delta t, t\right)=f_{a}(\boldsymbol{x}, t)-\frac{\Delta t}{\tau_{f}}\left[f_{a}(\boldsymbol{x}, t)-f_{a}^{\mathrm{eq}}(\boldsymbol{x}, t)\right]
$$

where $\Delta t$ is a constant increase in time and $\boldsymbol{x}+\boldsymbol{e}_{a} \Delta t$ denotes the position of the neighboring $\boldsymbol{x}$ in the direction of the velocity $\boldsymbol{e}_{a}$. The kinematic viscosity $v$ is defined by

$$
v=c_{s}^{2}\left(\tau_{f}-\frac{\Delta t}{2}\right)
$$

The Reynolds number is a primary dimensionless parameter for the behavior of all Newtonian fluids and is given by the ratio of the inertia and viscous forces:

$$
R e=\frac{L U}{v}
$$

where $U$ and $L$ are the characteristic velocity and length scales of the flow, respectively. Here, the differential fluid pressure $\Delta p_{\text {real }}$ in real dimensions can be calculated as follows:

$$
\Delta p_{\text {real }}=\frac{\left(\rho_{0} U^{2}\right)_{\text {real }}}{\rho_{0} U^{2}} \Delta p
$$

The intrinsic permeability from LBM can be calculated by using $\Delta p_{\text {real }}$ and the discharge from Eqs. (1), (2), and (18).

To compare the experimental results and LBM simulation, the appropriate parameters needed to be determined. The CT images had $300^{3}$ voxels with a $5 \mu \mathrm{m}$ resolution. The calculation area of LBM was $300^{3}$ lattice cells on a Cartesian axis with lattice spacings of $\Delta x=\Delta y=\Delta z=1$. The incremental increase in time $\Delta t$ was set to 1.0 for the calculation procedure. The simulations were run at $R e=2$ to be close to the experimental differential head, and additional analysis was performed at $R e=0.1$. The boundary conditions for $f_{a}$ of the moving particles were set as follows: For nonslip solid lattice cells, a bounce-back condition was applied to the particles moving with $\boldsymbol{e}_{a}$ from the fluid side to the solid side. These particles returned to the fluid side in the opposite direction of $-\boldsymbol{e}_{a}$. On the inflow and outflow boundaries, the particles coming into the calculation area from the outside were assumed to be in local equilibrium state according to the outside pressure and fluid velocity.

\section{Results}

\subsection{Experimental Results}

Equations (1) and (2) were used to calculate the intrinsic permeability in the directions normal and parallel to the bedding (Fig. 6). The permeability experiments were conducted three times for each direction. The average intrinsic permeability in the direction normal to the bedding $\left(k^{\perp}\right)$ was $1.03 \times 10^{-14} \mathrm{~m}^{2}$, while it was $8.76 \times 10^{-14} \mathrm{~m}^{2}$ in the parallel direction $\left(k^{\|}\right)$. 
Fig. 6 Amount of for specimens in the constant pressure permeability experiment. The experiment was conducted three times, and the results were averaged to determine the permeability in each direction

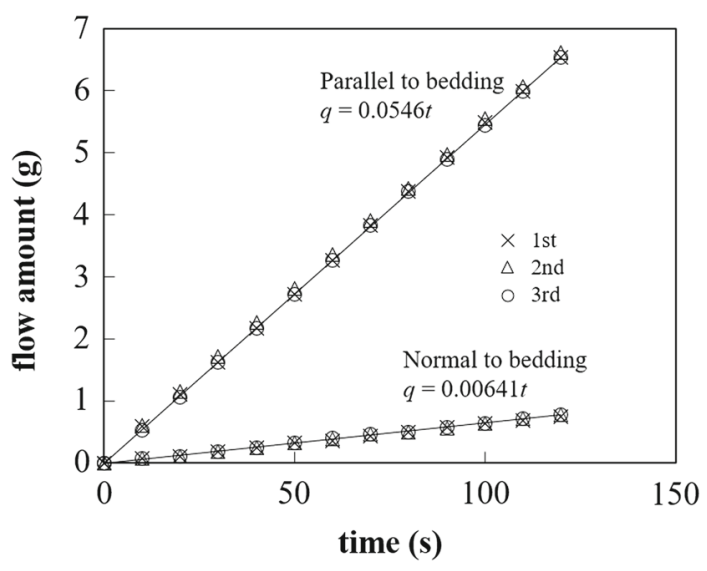

Figure 7 shows the results of the diffusivity experiments. The effective diffusion coefficient for $\mathrm{NaCl}$ was calculated from its linear relationship with $\ln \left(\Delta C / \Delta C_{0}\right)$, as given in Eq. (3). The effective diffusion coefficient in the directions parallel $\left(D_{\mathrm{e}}^{\|}\right)$and normal $\left(D_{\mathrm{e}}^{\perp}\right)$ to the bedding was $4.84 \times 10^{-11}$ and $3.49 \times 10^{-11} \mathrm{~m}^{2} / \mathrm{s}$, respectively. The anisotropy ratios for the intrinsic permeability and diffusion coefficient (i.e., $k^{\|} / k^{\perp}$ and $D_{\mathrm{e}}^{\|} / D_{\mathrm{e}}^{\perp}$ ) were estimated to be 8.50 and 1.39 , respectively. Table 1 presents the results obtained via the experimental procedure and LBM modeling (described in the subsequent sections). The difference in the anisotropy ratios suggests that a pore structure with a preferential orientation affects anisotropies of the permeability and diffusivity differently.

\subsection{CT Analysis}

Figure 8a shows the CT pore volume data of Berea sandstone for a volume of $1.5 \mathrm{~mm}^{3}$ $\left(300^{3}\right.$ voxels). Figure $8 \mathrm{~b}-\mathrm{d}$ shows $2-\mathrm{D}$ image cross sections in the $X, Y$, and $Z$ directions. The bulk density of the bedding was higher than that outside the bedding. Therefore, the bedding showed that the pores were less interconnected in the bedding plane. Kim et al. (2016) compared the porosity inside and outside the bedding of the Berea sandstone by using micro-focus X-ray CT. They observed that the zone outside the bedding has more pores than the zone inside the bedding and that the pores are more interconnected in the zone outside the bedding.

The tortuosity from one end face to the opposite side can be calculated from the medial axis (Fig. 5) of the 3-D CT image using Eq. (5). Figure 9 shows the tortuosity distribution in three directions. The tortuosity was greatest in the $\mathrm{Z}$ direction.

Figure 10 depicts the cross-sectional porosity change along the perpendicular $(Z)$ and parallel $(Y)$ directions to the bedding with pressure distribution from the LBM results. The Berea sandstone exhibited significant cross-sectional porosity changes in the $Z$ direction. The cross-sectional porosity was at its minimum of $7.87 \%$ at a distance of $0.6 \mathrm{~mm}$ from the end surface; however, it increased to $27.58 \%$ where the section passed through narrow pore spaces. In the $Y$ direction, however, the cross-sectional porosity was relatively consistent with the minimum and maximum cross-sectional porosities being $14.10 \%$ and $23.52 \%$, respectively. This directional anisotropic trend confirmed the characteristic pore geometry due to beach sedimentation (Fig. 2). 

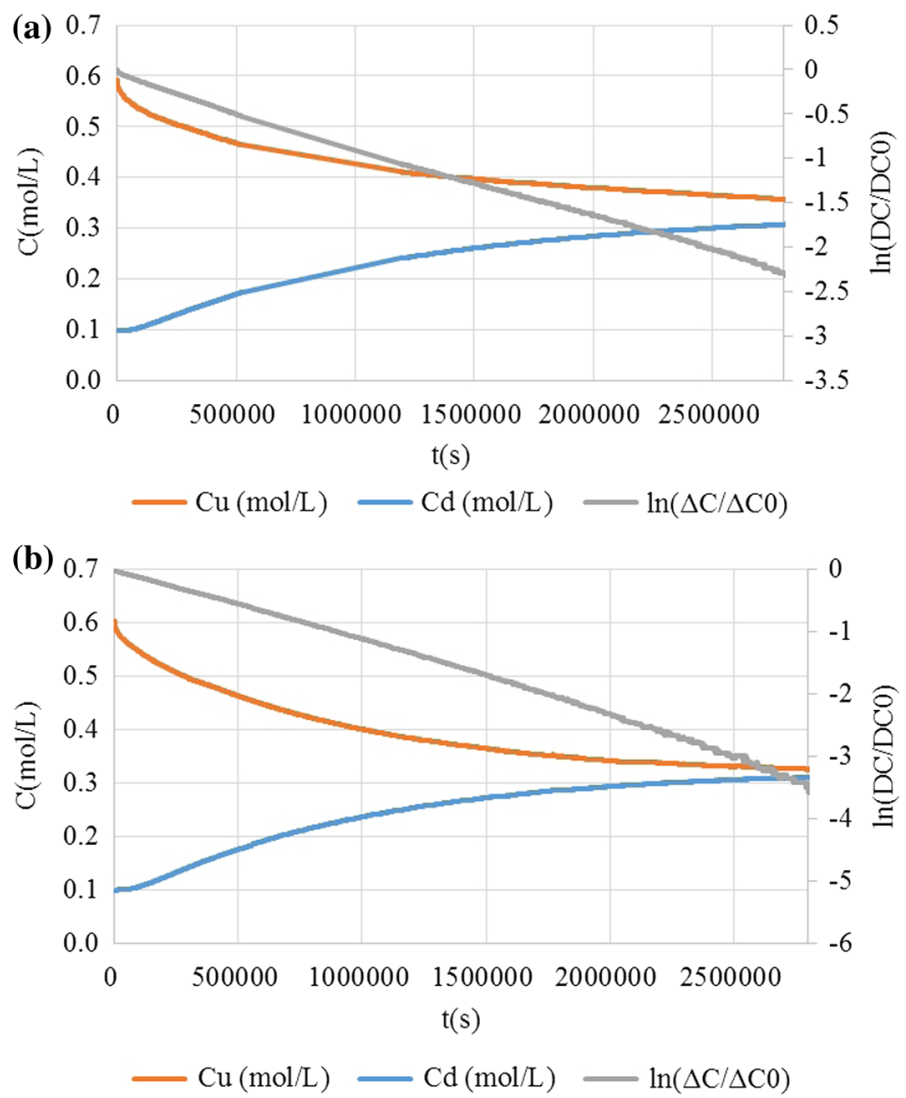

Fig. 7 Results of the diffusivity test. The $\mathrm{NaCl}$ concentrations of the reservoir solutions were measured in the directions a parallel and $\mathbf{b}$ perpendicular to the bedding

The model results for the median tortuosity $\left(\tau^{\|}=1.8\right.$ and $\left.\tau^{\perp}=1.95\right)$, constrictivity $\left(\delta_{\mathrm{g}}^{\|}=0.761\right.$ and $\left.\delta_{\mathrm{g}}^{\perp}=0.757\right)$, porosity $(\varphi=18.29 \%)$, and tracer diffusivity in bulk liquid water $\left(D_{\mathrm{w}}=2.015 \times 10^{-9} \mathrm{~m}^{2} / \mathrm{s}\right)$ were used in Eq. (4) to obtain the effective diffusivities in two perpendicular directions. The results for the parallel and normal directions to the bedding plane were $8.63 \times 10^{-11}$ and $7.35 \times 10^{-11} \mathrm{~m}^{2} / \mathrm{s}$, respectively, which are slightly higher than those obtained via the experimental procedure. The model of the diffusivity anisotropy is affected by tortuosity anisotropy in the case of using Eq. (4).

\subsection{LBM Results}

The LBM results for the steady-state flow after 500,000 time steps are presented here. The differential pressure calculated from Eq. (18) was $4.5 \mathrm{kPa}$, which is sufficiently close to the experimental condition $(3.0 \mathrm{kPa})$. Figure 10 shows the relation between the cross-sectional porosity and pressure distribution in the directions parallel and normal to the bedding. The pressure distribution in the normal direction to the bedding showed a sudden decrease at low-porosity areas. Figure 11 shows the typical fluid pressure distribution of the LBM flow 
Table 1 Permeability and diffusivity values obtained by experimental results and models
An isotropic ratio

\begin{tabular}{lll}
\hline \multicolumn{2}{l}{ Experimental results } & \\
$K^{\|}$ & $8.76 \times 10-14\left(\mathrm{~m}^{2}\right)$ & 8.50 \\
$K^{\perp}$ & $1.03 \times 10-14\left(\mathrm{~m}^{2}\right)$ & \\
$D_{\mathrm{e}}^{\|}$ & $4.84 \times 10-11\left(\mathrm{~m}^{2} / \mathrm{s}\right)$ & 1.39 \\
$D_{\mathrm{e}}^{\perp}$ & $3.49 \times 10-11\left(\mathrm{~m}^{2} / \mathrm{s}\right)$ & \\
$M o d e l$ & & \\
$K^{\|}$ & $4.26 \times 10-13\left(\mathrm{~m}^{2}\right)$ & 8.50 \\
$K^{\perp}$ & $5.01 \times 10-14\left(\mathrm{~m}^{2}\right)$ & \\
$D_{\mathrm{e}}^{\|}$ & $8.63 \times 10-11\left(\mathrm{~m}^{2} / \mathrm{s}\right)$ & 1.17 \\
$D_{\mathrm{e}}^{\perp}$ & $7.35 \times 10-11\left(\mathrm{~m}^{2} / \mathrm{s}\right)$ & \\
\hline
\end{tabular}
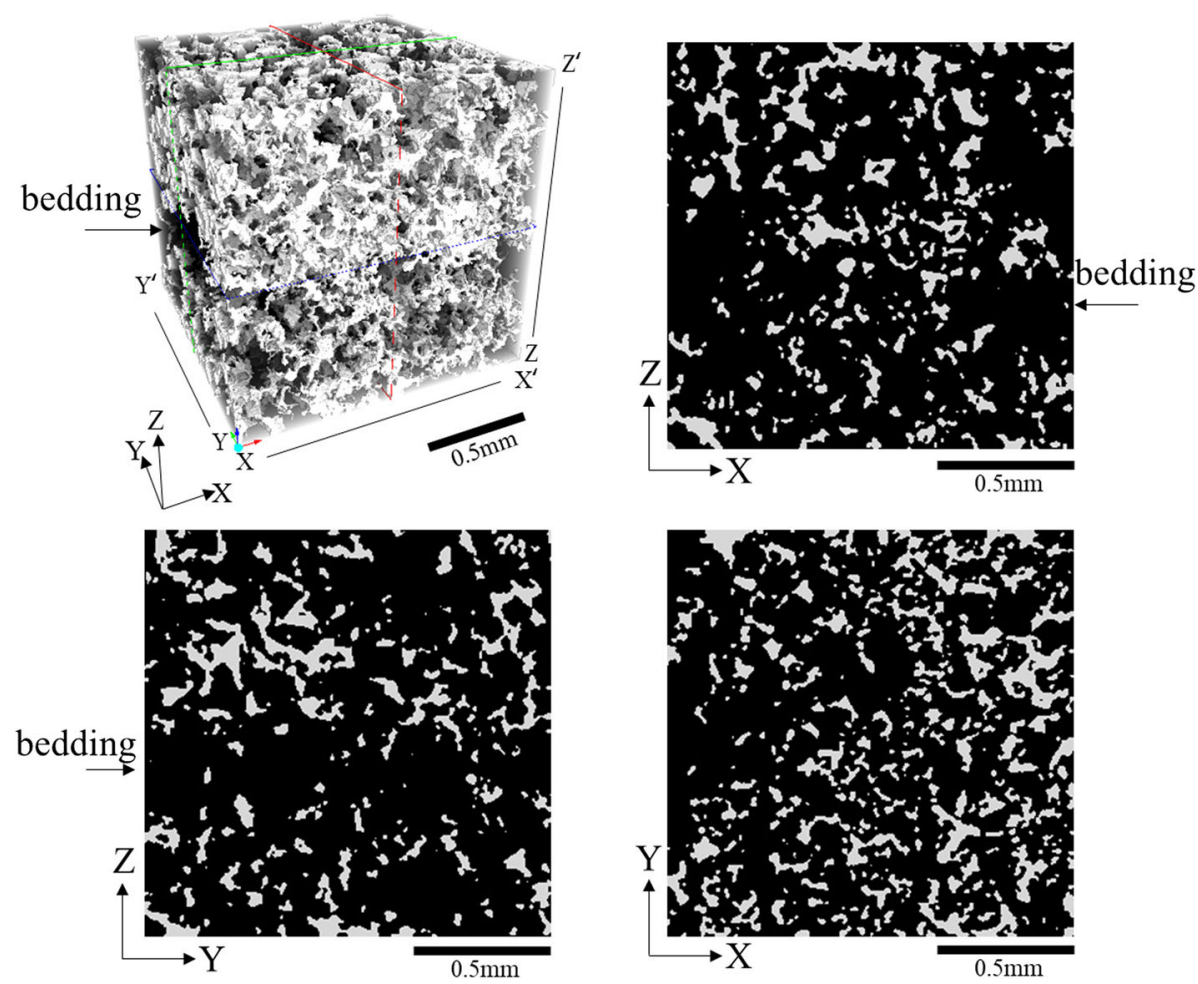

Fig. 8 Pore structure of Berea sandstone $(1.5 \mathrm{~mm} \times 1.5 \mathrm{~mm} \times 1.5 \mathrm{~mm})$. a The light and dark areas correspond to grain and pore spaces, respectively. b-d The light and dark areas correspond to pore spaces and grain, respectively

in the direction parallel to the bedding $\left(Y-Y^{\prime}\right)$. The fluid pressure gradually decreased with distance from the inflow boundary.

Figure 12 depicts a representative section cut from the 3-D LBM simulation results. The rock pores are not connected in the 2-D image because the pore structure is very intricate. 


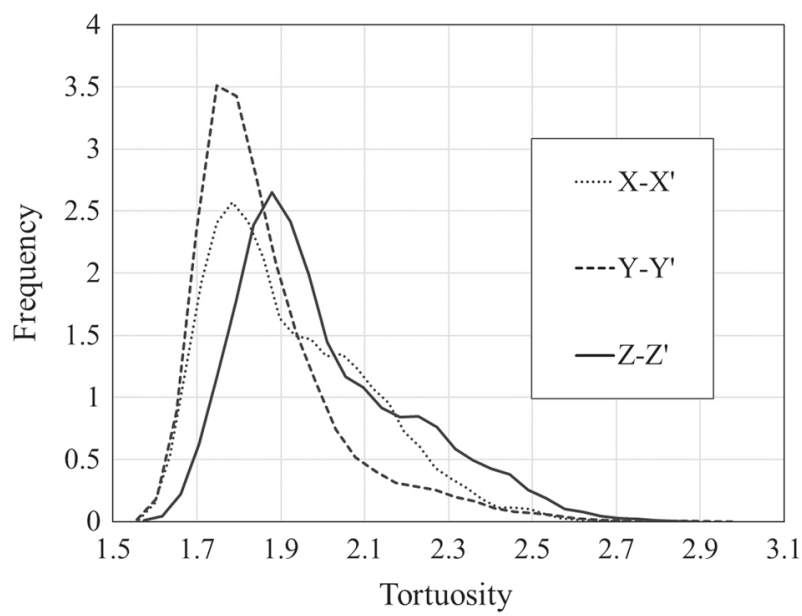

Fig. 9 Tortuosity distribution in three directions. The $X-X^{\prime}$ and $Y-Y^{\prime}$ directions are parallel to the bedding, and the $Z-Z^{\prime}$ direction is normal to the bedding

Figures $12 \mathrm{~b}-\mathrm{f}$ is acquired using the maximum intensity projection, which enabled the maximum flow velocity and/or fluid pressure to be illustrated as 2-D front view figures for a $0.15 \mathrm{~mm}$ (30 pixels) thick plate containing 3-D pore structures. The bedding with the minimum cross-sectional porosity in the $Z$ direction was at $0.6 \mathrm{~mm}$ from the bottom. The flow velocity in the $\mathrm{Y}$ direction was relatively uniform (Fig. 12c). The pressure distribution in the $Y$ direction (Fig. 12d) showed that the pressure gradually decreased with distance from the inflow boundary. On the other hand, a high flow rate at low-porosity areas was noted in the $\mathrm{Z}$ direction (Fig. 12e). The pressure distribution results in the $Z$ direction (Fig. 12f) showed a sudden decrease at low-porosity areas, for which the numeric data are shown in Fig. 10b. Hence, the advection flow is restricted by a bottleneck effect resulting from low cross-sectional porosity in the direction normal to the bedding.

The steady-state intrinsic permeabilities in the $Z\left(k_{\mathrm{LBM}}^{\perp}\right)$ and $\mathrm{Y}\left(k_{\mathrm{LBM}}^{\|}\right)$directions were $5.01 \times 10^{-14}$ and $4.26 \times 10^{-13} \mathrm{~m}^{2}$, respectively. The anisotropy ratio of the permeability $\left(k_{\mathrm{LBM}}^{\|} / k_{\mathrm{LBM}}^{\perp}\right)$ was 8.50 .

\section{Discussion}

\subsection{Diffusivity Anisotropy Due to the Pore Structure in the Bedding}

In this study, the experimental diffusivities were acquired as $D_{\mathrm{e}}^{\|}=4.84 \times 10^{-11} \mathrm{~m}^{2} / \mathrm{s}$ and $D_{\mathrm{e}}^{\perp}=3.49 \times 10^{-11} \mathrm{~m}^{2} / \mathrm{s}$. For reference, the diffusivity of $\mathrm{Cl}^{-}$in Berea sandstone is $1.03 \times$ $10^{-10} \mathrm{~m}^{2} / \mathrm{s}$ (Yokoyama and Nakashima 2005), which is approximately the same as the value calculated from the CT data. The relatively low diffusivities in the normal direction to the bedding have been reported in many other studies. Consequently, the results of this study confirm the diffusivity anisotropy due to anisotropic pore structures in the bedding.

One possible reason for the difference between the calculated and experimental results is the overestimation of the pore size. When binarization is carried out according to the porosity, the volume of nanopores with radii of under $5 \mu \mathrm{m}$ is replaced with that of pores having radii 
(a) Parallel to bedding

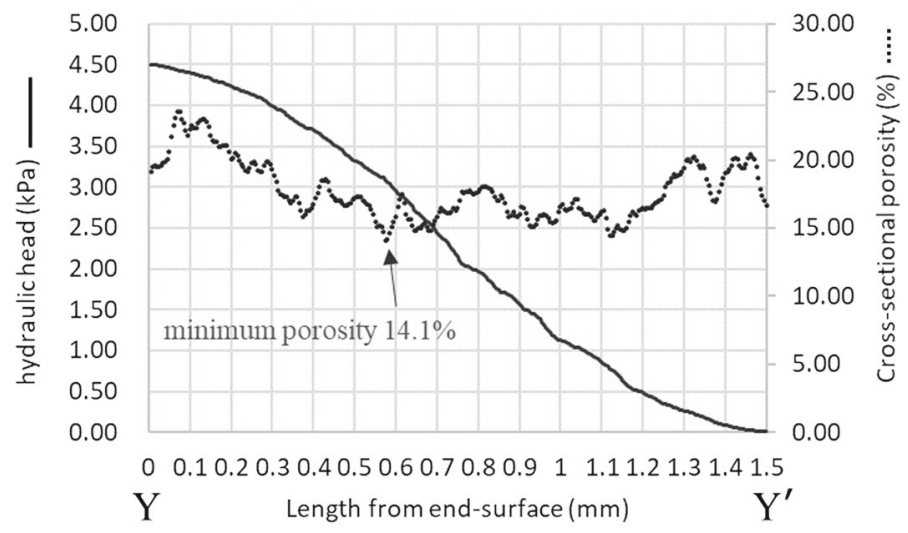

(b) Normal to bedding

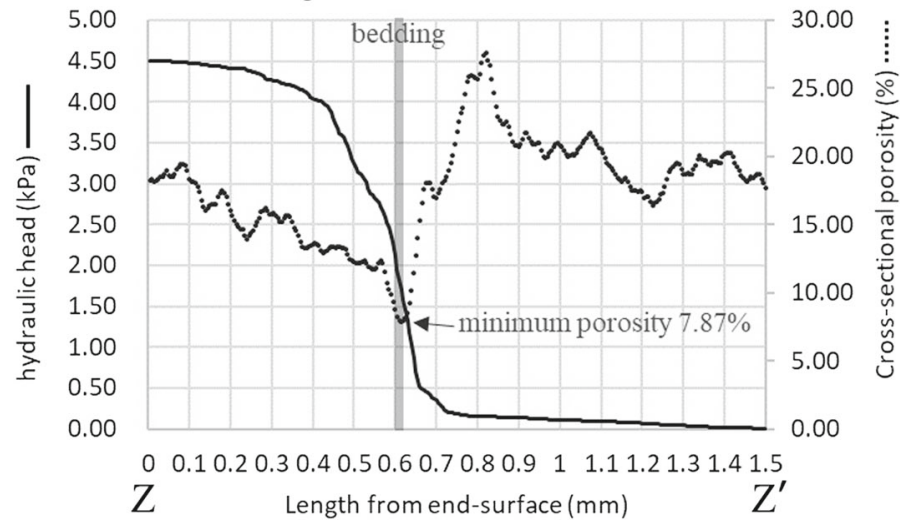

Fig. 10 Cross-sectional porosity of the sample in the directions parallel $\left(Y-Y^{\prime}\right)$ and normal $\left(Z-Z^{\prime}\right)$ to the bedding plane

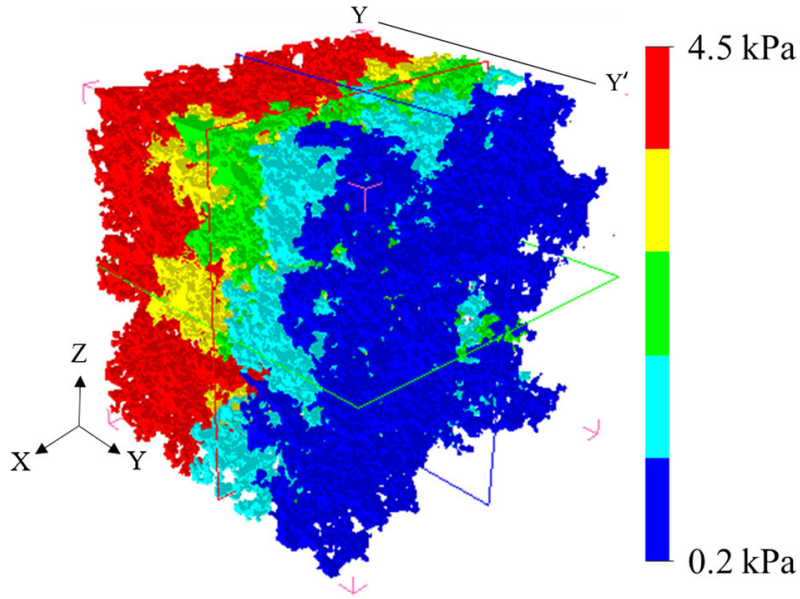

Fig. 11 Fluid pressure distribution of the LBM flow from the $X-Z$ plane 

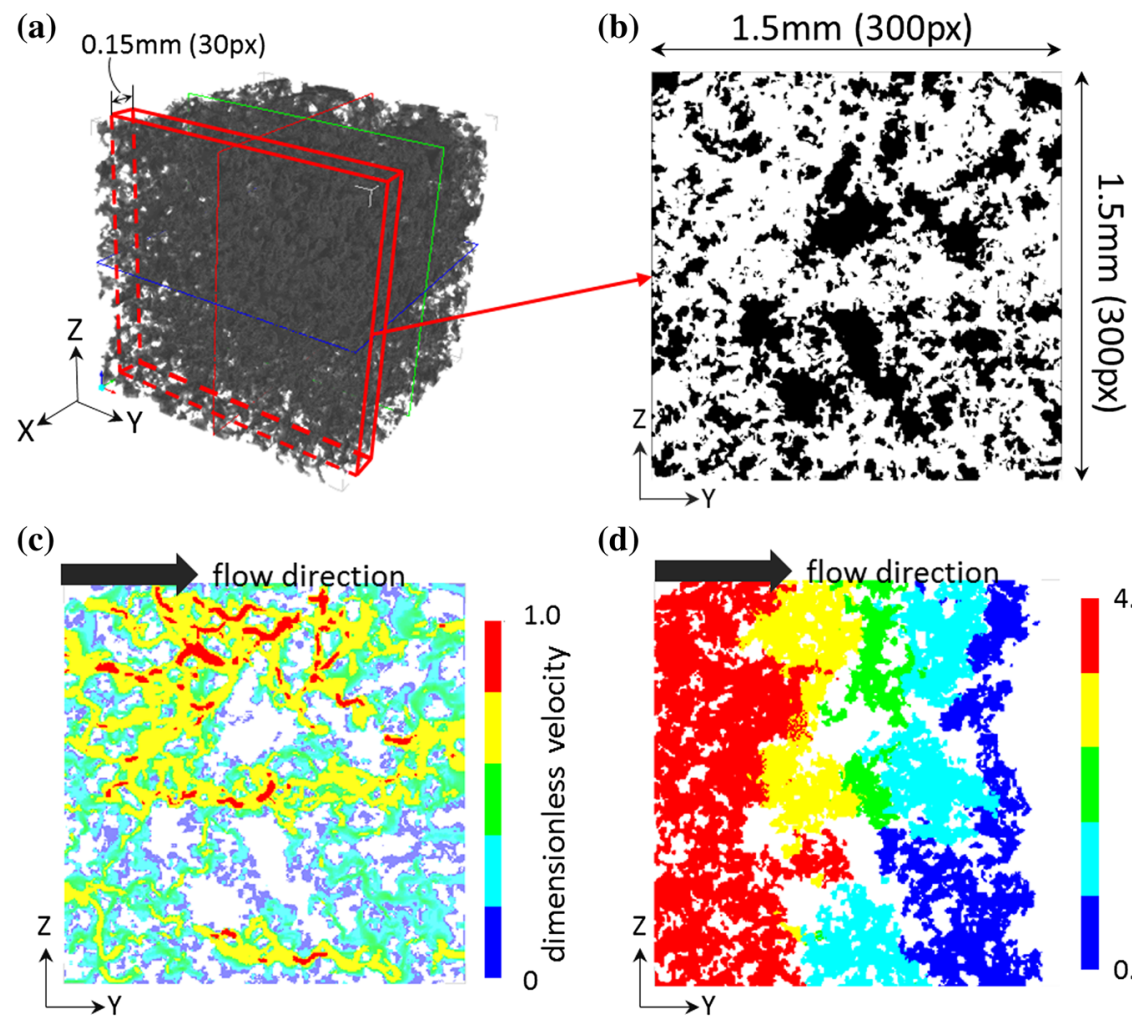

(d)

(e)

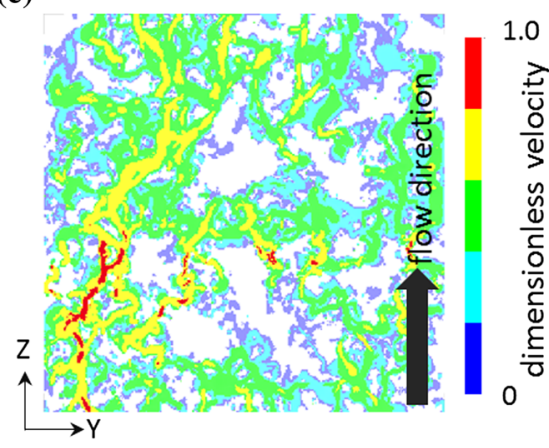

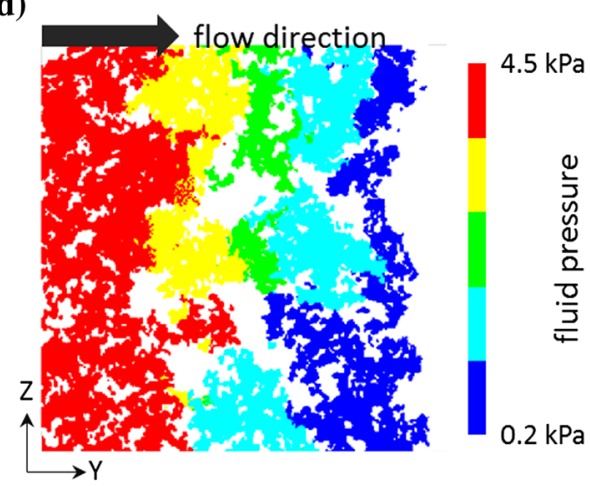

(f)

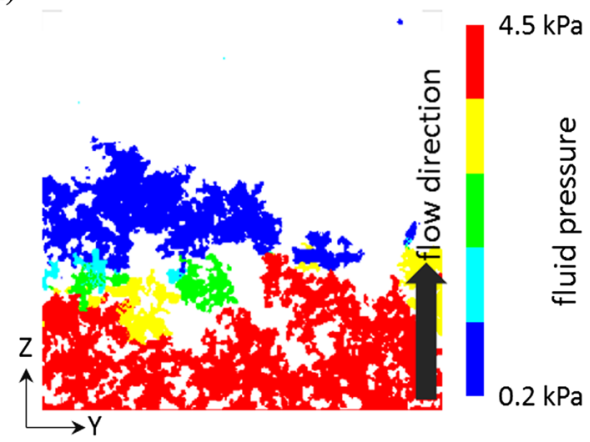

Fig. 12 LBM results: a pore structure taken from X-ray CT, b pore structure cut from the 3D pore structure with a $0.15 \mathrm{~mm}$ thickness. Platen figures cut from the $3 \mathrm{D} \mathrm{LBM}$ results applied to the cubic figure: flow velocity and pressure distribution of the pore structure for the directions $\mathbf{c}, \mathbf{d}$ parallel to the bedding and $\mathbf{e}, \mathbf{f}$ normal to the bedding as shown by the maximum intensity projection

of over $5 \mu \mathrm{m}$, which extends the pore structure. Further high-resolution analyses and/or the application of additional simulation for the diffusivity are required to arrive at a more detailed explanation. 


\subsection{Permeability Anisotropy Due to the Bedding Plane}

The permeability was also higher in the parallel direction to the bedding than in the normal direction. In addition, the permeabilities from the LBM simulation were higher than the corresponding experimental values, which may stem from the resolution problem. However, Peng et al. (2014) showed two micro-focus CT images of Berea sandstone that were binarized using the Kriging method with resolutions of 1.85 and $5.92 \mu \mathrm{m} /$ voxel, which returned nearly identical permeabilities. This indicates that $5.92 \mu \mathrm{m}$ is a sufficiently high resolution to capture the main flow paths for this rock and that smaller pores need not always be resolved. The permeability ratios in the directions perpendicular and parallel to the bedding were almost the same as those in the experimental results. Thus, the LBM simulation is considered to be applicable for evaluating the permeability anisotropy.

The permeability experiment was conducted at a differential pressure of $3 \mathrm{kPa}$. Because Berea sandstone has high permeability, the flow rate through the specimen was probably fast enough to allow for a non-Darcy flow. As indicated in Fig. 12, the advective flow was restricted by the bottleneck effect because of the low cross-sectional porosity in the normal direction to the bedding. The combination of the bottleneck pore structure, fast flow velocity, and opening out after narrow pore spaces resulted in high $R e$ numbers and turbulent flow. In the experiments, the discharge values in the directions parallel and normal to the bedding were $5.46 \times 10^{-6} \mathrm{~m}^{3} / \mathrm{s}$ and $6.41 \times 10^{-7} \mathrm{~m}^{3} / \mathrm{s}$, respectively; the kinematic viscosity of water at $40{ }^{\circ} \mathrm{C}$ was $6.58 \times 10^{-7} \mathrm{~m}^{2} / \mathrm{s}$; the characteristic length was $2.64 \times 10^{-2} \mathrm{~m}$ which was assumed to be the diameter of the total pore spaces of the cross section with a diameter of $50 \mathrm{~mm}$; and the porosity was $18.29 \%$. $R e^{\|}$and $R e^{\perp}$ were 5.50 and 0.58 , respectively. In real flow, $R e$ is dependent on the size of each pore. Even though it is difficult to determine the value of $R e$ in the experiment, $R e=2$ in the LBM simulation represents an appropriate value, which is of the order of the experimental Re. Using 2-D LBM for a virtual pore structure, Sato and Takeda (2016) pointed out that the fluid pressure mainly drops at pore throats. Therefore, turbulent flow is unlikely to occur under low flow rate conditions, and hence, the permeability anisotropy is reduced. They conducted their LBM simulation at $R e=0.1$, which would not allow turbulent flow to occur. In the case of LBM simulation for pore structure of Berea sandstone at $R e=0.1$, the results of $k^{\|}=1.37 \times 10^{-12} \mathrm{~m}^{2}$ and $k^{\perp}=3.23 \times 10^{-13} \mathrm{~m}^{2}$ were derived. The anisotropic permeability ratio $\left(k^{\perp} / k^{\|}\right)$was 4.24 , which is smaller than the permeability ratio of 8.50 at $R e=2$. Clearly, the bottleneck effect is reduced under the no turbulent flow condition due to a low flow velocity.

\subsection{Comparison of the Permeability and Diffusivity Anisotropies}

In the experimental results, the anisotropy ratios of the permeability $\left(k^{\perp} / k^{\|}\right)$and diffusivity $\left(D_{\mathrm{e}}^{\perp} / D_{\mathrm{e}}^{\|}\right)$were 8.50 and 1.39 , respectively. This indicates that the pore structure anisotropy of the bedding had a stronger effect on the permeability than on the diffusivity. As shown in Fig. 12, the flow velocity was restricted by the bottleneck effect because of the low crosssectional porosity $(7.87 \%)$ in the normal direction to the bedding. In the parallel direction to the bedding plane, the minimum cross-sectional porosity was $14.1 \%$, and a gradual decrease in fluid pressure was apparent. The diffusivity in each direction was calculated by using tortuosity with porosity. The calculated diffusivity ratio $\left(D_{\mathrm{e}}^{\perp} / D_{\mathrm{e}}^{\|}\right)$was less than the experimental results. This can be attributed to expanding a specific pore structure to the experimental sample block which is similar to extrapolating one bedding plane to a block layer. 
In the case of ionic diffusion, ions can pass through the existing narrow pore spaces. This made the anisotropic diffusivity ratio smaller than the anisotropic permeability ratio. Peng et al. (2014) showed that $5.92 \mu \mathrm{m}$ is a sufficiently high resolution to capture the main flow paths for the rock and that the smaller pores need not always be resolved. For ionic diffusion, however, Kikuchi et al. (2010) suggested that $\mathrm{Cl}^{-}$is restricted to pore radii of $6 \mathrm{~nm}$ or higher. This work focused on the separate phenomena on permeability and diffusion parameters. Further high-resolution simulations are needed to shed light on the limit values for the permeability and diffusivity.

\section{Conclusions}

In this study, the effect of pore structure on the transport parameters' anisotropy in Berea sandstone, whose grain arrangement is dominated by sedimentation, was scrutinized. Implementing both experimental and numerical modeling approaches, the anisotropy ratios for the intrinsic permeability $\left(k^{\perp} / k^{\|}\right)$and effective diffusion coefficient $\left(D_{\mathrm{e}}^{\perp} / D_{\mathrm{e}}^{\|}\right)$were estimated as 8.50 and 1.39 , respectively. The difference in the ratios suggests that a preferentially oriented pore structure affects the permeability and diffusivity anisotropies in a meaningful manner. The advective flow in the normal and parallel directions to the bedding was simulated by LBM using a 3-D pore structure obtained from X-ray CT. The flow simulation results showed that the fluid pressures decreased primarily at areas of minimum cross-sectional porosity in the normal direction to the bedding. In the parallel direction to the bedding, the pressure gradually decreased with distance from the inflow boundary. The anisotropic diffusivity ratio was less than the anisotropic permeability ratio because, in the case of $\mathrm{NaCl}$ diffusion, ions can pass through very narrow pore spaces. The proposed bulk constrictivity in this study is the corresponding value of a sample's specific pore structure on one bedding plane. Based on the experimental results, CT analysis, and LBM simulation, expanding one specific pore structure to an experimental sample of one block can be assumed to be similar to expanding one bedding plane to one block layer. The results suggest that the minimum cross-sectional porosity, which affects the permeability anisotropy by physically restricting moving elements, is still too large to induce anisotropic ionic diffusion. Such observations can pave the way for further investigation on the permeability and diffusivity anisotropy effects in reservoir modeling projects for geologic disposal of highly contaminant waste, hydrocarbon recovery, or storing $\mathrm{CO}_{2}$ in porous subsurface formations, rendering more comprehensive design considerations.

Acknowledgements We are grateful to the referees for helpful comments.

Open Access This article is distributed under the terms of the Creative Commons Attribution 4.0 International License (http://creativecommons.org/licenses/by/4.0/), which permits unrestricted use, distribution, and reproduction in any medium, provided you give appropriate credit to the original author(s) and the source, provide a link to the Creative Commons license, and indicate if changes were made.

\section{References}

ASTM D2434-68 (2006).: Standard Test Method for Permeability of Granular Soils (Constant Head) (Withdrawn 2015). ASTM International, West Conshohocken, PA (2006). www.astm.org 
Auzerais, F.M., Dunsmuir, J., Ferreol, B.B., Martys, N., Olson, J., Ramakrishnan, T.S., Rothman, D.H., Schwartz, L.M.: Transport in sandstone: a study based on three dimensional microtomography. Geophys. Res. Lett. 23(7), 705-708 (1996)

Benzi, R., Succi, S., Vergassola, M.: The lattice Boltzmann equation: theory and applications. Phys. Rep. 222, 145-197 (1992)

Chen, S., Doolen, G.D.: Lattice Boltzmann method for fluid flows. Annu. Rev. Fluid Mech. 30, 329-364 (1998)

Clavaud, J.B., Maineult, A., Zamora, M., Rasolofosaon, P., Schlitter, C.: Permeability anisotropy and its relations with porous medium structure. J. Geophys. Res. 113, B01202 (2008). https://doi.org/10.1029/ 2007jb005004

Farver, J.R., Yund, R.A.: Oxygen bulk diffusion measurements and TEM characterization of a natural ultramylonite: implications for fluid transport in mica-bearing rocks. J. Metamorph. Geol. 17, 669-683 (1999)

Fujii, Y., Takahashi, M.: Geology, sedimentary environment and physical properties of Berea sandstone. J. Jpn. Soc. Eng. Geol. 56(3), 105-109 (2015). (In Japanese)

Gao, J., Xing, H., Rudolph, V., Li, Q., Golding, S.D.: Parallel lattice Boltzmann computing and applications in core sample fracture evaluation. Transp. Porous Med. 107, 65-77 (2015)

Genty, A., Gueddani, S., Dymitrowska, M.: Computation of saturation dependance of effective diffusion coefficient in unsaturated argillite micro-fracture by lattice Boltzmann method. Porous Med., Transp (2017). https://doi.org/10.1007/s11242-017-0826-z

He, X., Luo, L.-S.: Lattice Boltzmann model for the incompressible Navier-Stokes equation. J. Stat. Phys. 88, 927-944 (1997)

Inamuro, T., Yoshino, M., Ogino, F.: Lattice Boltzmann simulation of flows in a three-dimentional porous structure. Int. J. Numer. Methods Fluids 29, 737-748 (1999)

Kikuchi, M., Suda, Y., Saeki, T.: Evaluation for ion transport in hardened cementitious paste by oxygen diffusion and chloride diffusion. Cem. Sci. Concr Technol 64, 346-353 (2010). (In Japanese)

Kim, K.Y., Zhuang, L., Yang, H., Kim, H., Min, K.-B.: Strength anisotropy of Berea sandstone: results of X-ray computed tomography, compression tests, and discrete modeling. Rock Mech. Rock Eng. 49, 1201-1210 (2016). https://doi.org/10.1007/s00603-015-0820-0

Lindquist, W.B., Lee, S.M., Coker, D.A., Jones, K.W., Spanne, P.: Medial axis analysis of void structure in three-dimensional tomographic images of porous media. J. Geophys. Res. 101(B4), 8297-8310 (1996)

Lindquist, W.B., Venkatarangan, A.: Investigation 3D geometry of porous media from high resolution images. Phys. Chem. Earth Part A 25(7), 593-599 (1999)

Misawa, M., Tiseanu, I., Hirashima, R., Koizumi, K., Ikeda, Y.: Oblique view cone beam tomography for inspection of flat-shape objects. Key Eng. Mater. 270, 115-1142 (2004)

Okabe, H., Blunt, M.J.: Prediction of permeability for porous media reconstructed using multiple-point statistics. Phys. Rev. E 70(066135), 1-10 (2004)

Onishi, J., Chen, Y., Ohashi, H.: Lattice Boltzmann simulation of natural convection in a square cavity. JSME Int. J. Ser. B Fluids Therm. Eng. 44, 53-62 (2001)

Peng, S., Marone, F., Dultz, S.: Resolution effect in X-ray microcomputed tomography imaging and small pore's contribution to permeability for a Berea sandstone. J. Hydrol. 510, 403-411 (2014)

Sato, M., Takeda, M.: Microstructure Effects on Anisotropy in Permeability and Diffusivity of Berea Sandstone, p. \#H51B-1470. American Geophysical Union, Fall General Assembly, New York (2016)

Takahashi, H., Seida, Y., Yui, M.: 3D X-ray CT and diffusion measurements to assess tortuosity and constrictivity in a sedimentary rock. Diffus. Fund. Org. 11, 1-11 (2009)

Takeda, M., Hiratsuka, T., Manaka, M., Finsterle, S., Ito, K.: Experimental examination of the relationships among chemico-osmotic, hydraulic, and diffusion parameters of Wakkanai mudstones. J. Geophys. Res. Solid Earth (2014). https://doi.org/10.1002/2013JB010421

Van Brakel, J., Heertjes, P.M.: Analysis of diffusion in macroporous media in terms of a porosity, a tortuosity and a constrictivity factor. Int. J. Heat Mass Transf. 17, 1093-1103 (1974)

Van Loon, L.R., Soler, J.L., Müller, W., Bradbury, M.H.: Anisotropic diffusion in layered argillaceous formations: a case study with opalinus clay. Environ. Sci. Technol. 38, 5721-5728 (2004)

Wolfrum, C., Lang, H., Moser, H., Jordan, W.: Determination of diffusion-coefficients based on Fick's second law for various boundary-conditions. Radiochem. Acta 44, 245-249 (1988)

Wan, Y., Pan, Z., Tang, S., Connel, L.D., Down, D.D., Camilleri, M.: An experimental investigation of diffusivity and porosity anisotropy of a Chinese gas shale. J. Nat. Gas Sci. Eng. 23, 70-79 (2015)

Yokoyama, T., Nakashima, S.: Diffusivity anisotropy in a rhyolite and its relation to pore structure. Eng. Geol. 80, 328-335 (2005)

Publisher's Note Springer Nature remains neutral with regard to jurisdictional claims in published maps and institutional affiliations. 